\title{
The CRASH report: emergency management dilemmas facing acute physicians in patients with pulmonary arterial hypertension
}

\author{
Laura C Price, ${ }^{1}$ Konstantinos Dimopoulos, ${ }^{1,2}$ Philip Marino, ${ }^{3,4}$ \\ Rafael Alonso-Gonzalez, ${ }^{1,2}$ Colm McCabe, ${ }^{1}$ Aleksander Kemnpy, ${ }^{1}$ Lorna Swan, ${ }^{2}$ \\ Maria Boutsikou, ${ }^{2}$ Ahmed Al Zahrani, ${ }^{2}$ Gerry J Coghlan, ${ }^{4}$ Benjamin E Schreiber, \\ Luke S Howard, ${ }^{5}$ Rachel Davies, ${ }^{5}$ Mark Toshner, ${ }^{6}$ Joanna Pepke-Zaba, ${ }^{6}$ \\ Alistair C Church, ${ }_{1}^{7}$ Andrew Peacock, ${ }^{7}$ Paul A Corris, ${ }^{8}$ James L Lordan, ${ }^{8}$ Sean Gaine, ${ }^{9}$ \\ Robin Condliffe, ${ }^{10}$ David G Kiely, ${ }^{10}$ Stephen John Wort ${ }^{1}$
}

For numbered affiliations see end of article.

\section{Correspondence to}

Dr Laura C Price, Consultant Respiratory Physician, National Pulmonary Hypertension Service, Royal Brompton Hospital, Sydney Street, London SW3 6NP, UK; laura.price@rbht.nhs.uk

Received 13 November 2016 Revised 6 July 2017 Accepted 17 July 2017

Published Online First 12 September 2017

\section{CrossMark}

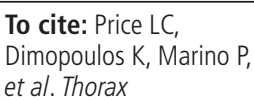

\section{ABSTRACT}

Treatment of acute emergencies in patients with pulmonary arterial hypertension (PAH) can be challenging. In the UK and Ireland, management of adult patients with $\mathrm{PAH}$ is centred in eight nationally designated pulmonary hypertension $(\mathrm{PH})$ centres. However, many patients live far from these centres and physicians in local hospitals are often required to manage PAH emergencies. A committee of physicians from nationally designated $\mathrm{PH}$ centres identified the 'most common' emergency clinical scenarios encountered in patients with PAH. Thereafter, a review of the literature was performed centred on these specified topics and a management approach was developed based on best available evidence and expert consensus. Management protocols were developed on the following PAH emergencies: chest pain (including myocardial ischaemia), right ventricular failure, arrhythmias, sepsis, haemoptysis ('CRASH'), as well as considerations relevant to surgery, anaesthesia and pregnancy. Emergencies are not uncommon in PAH. While expertise in PAH management is essential, all physicians involved in acute care should be aware of the principles of acute management of PAH emergencies. A multidisciplinary approach is necessary, with physicians from tertiary $\mathrm{PH}$ centres supporting care locally and planning safe transfer of patients to $\mathrm{PH}$ centres when appropriate.

\section{INTRODUCTION}

Pulmonary arterial hypertension (PAH) represents a group of conditions, which share the haemodynamic definition of a mean pulmonary artery pressure of greater than or equal to $25 \mathrm{~mm} \mathrm{Hg}$ and a pulmonary artery wedge pressure of less than or equal to $15 \mathrm{~mm} \mathrm{Hg}$. PAH comprises group 1 of the international classification of pulmonary hypertension $(\mathrm{PH}){ }^{1}$ The hallmark pathogenic abnormality in PAH is progressive remodelling of the arterial resistance vessels in the lung, leading to increased pulmonary vascular resistance (PVR). This, in turn, leads to an increase in right ventricular (RV) afterload, which, if untreated, undergoes well described morphological changes leading to RV failure and premature death. The prevalence of PAH is estimated at $15-52$ per million. ${ }^{2}$ Severe $\mathrm{PAH}$, therefore, remains a rare, although devastating disease. Other forms of $\mathrm{PH}$ include $\mathrm{PH}$ due to left-sided heart disease (group $2 \mathrm{PH}$ ), $\mathrm{PH}$ due to respiratory disease and/or hypoxia (group 3 ) and chronic thromboembolic PH (CTEPH, group 4). ${ }^{1}$ Similar physiological principles in managing acute medical emergencies may apply, although most available evidence is in PAH due to its clearly defined phenotype and response to PAH therapies.

Patients with $\mathrm{PAH}$ are best managed at specialist centres. ${ }^{3}$ In the UK and Ireland, there are currently eight adult commissioned centres. ${ }^{4}$ However, the majority of patients with $\mathrm{PAH}$ do not live near tertiary $\mathrm{PH}$ centres and, when acutely unwell, may present to local hospitals and emergency services. Where logistic or medical reasons preclude immediate transfer to a specialist centre, emergency treatment must be provided locally.

This review describes the most common emergencies in patients with $\mathrm{PAH}$, including chest pain, RV failure, arrhythmias, sepsis, haemoptysis ('CRASH'), as well as considerations relevant to surgery, anaesthesia and pregnancy. The outcome from all 'CRASH scenarios' ultimately depends on the severity of RV dysfunction and failure, consideration of which is key to management at every step. We describe best management of these emergencies based on the available literature, current recommendations and expert opinion.

\section{METHODS}

A committee of UK PH physicians agreed on the most common medical emergencies in PAH. Two independent researchers reviewed the literature using agreed scenarios in $\mathrm{PAH}$, with search terms being PAH and the listed CRASH emergencies, non-cardiac surgery, anaesthesia or pregnancy using EMBASE, MEDLINE, PubMed, Scopus, Cochrane and clinicaltrials. gov, without limits on publication time or genre. Reviews, clinical trials, case reports, and conference abstracts and recommendation/ guideline papers were collected. The literature 
Box 1 Potential causes of chest pain in patients with pulmonary arterial hypertension (PAH)

\section{Ischaemic:}

- Atherosclerotic coronary artery disease

- Compression of the main stem of the left coronary artery due to dilatation of pulmonary artery

- Tachyarrhythmia (often supraventricular), leading to reduced diastolic time

\section{Non-ischaemic:}

- Pulmonary embolism, pulmonary infarct, pneumonia, pericarditis (including lupus related)

- Pulmonary arterial dissection or rupture with/without tamponade

Meanwhile, always assess for 'common' usual causes of chest pain applicable to all patients.

was critically reviewed and management recommendations were formulated.

\section{RESULTS}

\section{Clinical dilemmas}

Management of chest pain in a patient with PAH

Chest pain is a relatively common symptom in patients with $\mathrm{PAH}$. In this setting, common causes of chest pain, such as coronary atherosclerosis, acute pulmonary embolism and arrhythmia are most likely and should always be excluded, while also considering PAH-related causes of chest pain, such as sub-endocardial RV ischaemia and, importantly, pulmonary artery dilatation causing compression of the left coronary artery (box 1). Under normal conditions, blood flow to the right coronary artery (RCA) occurs throughout the cardiac cycle. In PAH, increased RV afterload impedes systolic flow to the RCA. ${ }^{5}$ Diastolic RCA flow may also be impaired due to increased right atrial (RA) pressure and, therefore, a reduced RA-aortic pressure gradient. Moreover, diastolic time is often compromised, particularly in patients with failing $\mathrm{RV}^{6}{ }^{6}$ and further tachyarrhythmia or hypotension may induce critical RV ischaemia.

Extrinsic left main stem coronary artery compression by a dilated pulmonary artery is increasingly described in the literature, ${ }^{7}$ presenting with chest pain and potentially catastrophic myocardial ischaemia ${ }^{8-10}$ (figure $1 \mathrm{H}$ ), especially in patients with an increased pulmonary artery (PA) diameter on $\mathrm{CT}^{7}$ Finally, pulmonary artery dissection and rupture are potential causes of chest pain and unexpected death in PAH: patients with a PA diameter exceeding $48 \mathrm{~mm}$ are at a 7.5 -fold increased risk of death $^{11}$ (figure $1 \mathrm{~A}-\mathrm{G}$ ).

Patients with significant $\mathrm{PAH}$ presenting with angina can be challenging to assess. In the acute setting, ECG changes should be interpreted by comparison to prior traces whenever possible, as right bundle branch block and right ventricular hypertrophy with associated ST-T wave changes are often present at baseline. Serial troponin levels should be measured and interpreted in the context of the clinical presentation and comorbidities, noting that troponin levels may even be elevated due to RV dysfunction in patients with stable disease at baseline. ${ }^{12}$ Evaluation of regional wall motion abnormalities on echocardiography should take into account changes relating to the underlying disease and RV overload (eg, deviation of the ventricular septum, RV dysfunction).
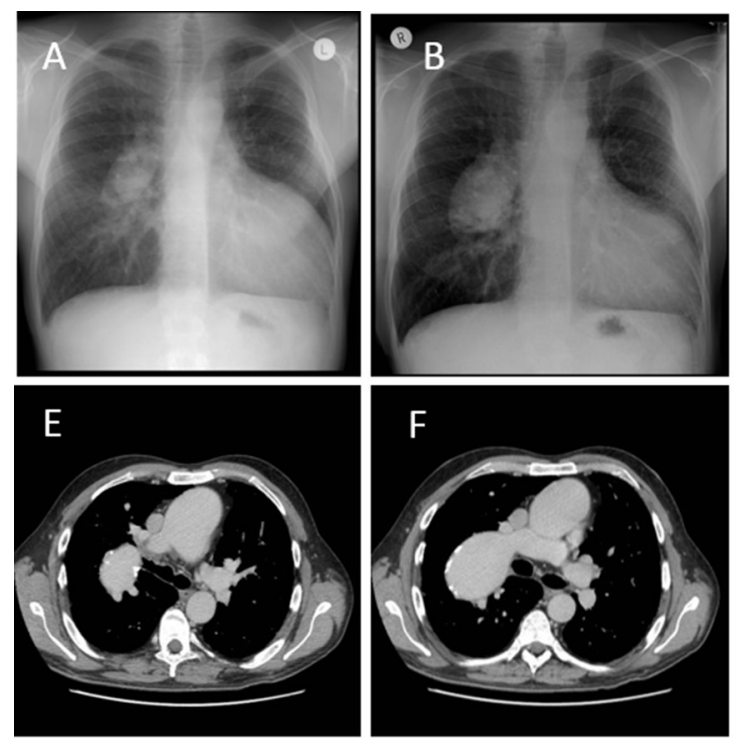
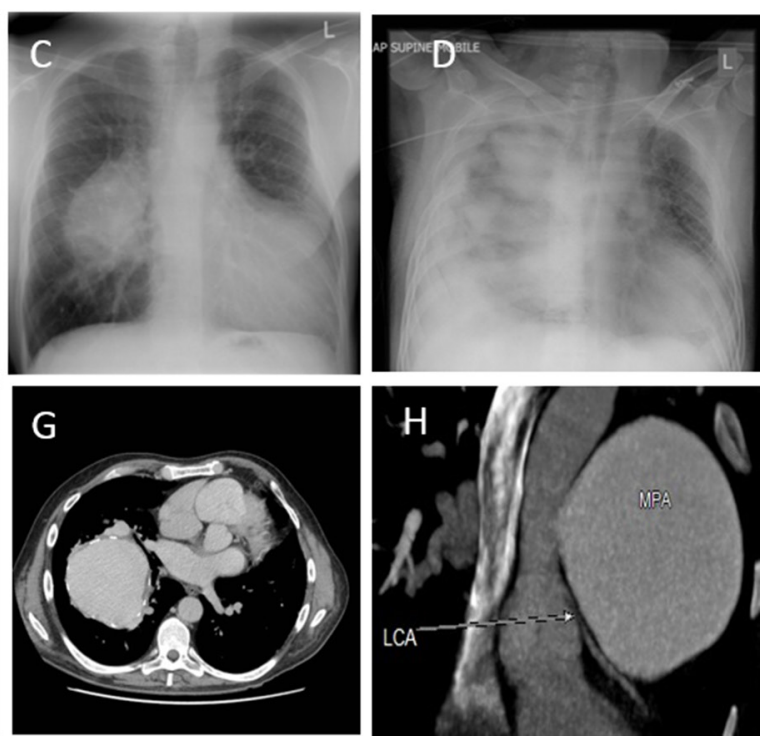

Figure 1 Massive haemoptysis $(A-G)$ and compression of coronary arteries in patients with PAH (H). Figures (A-G) show massive proximal dilatation of pulmonary trunk in pulmonary arterial hypertension (PAH) with Eisenmenger syndrome due to a ventricular septal defect. Chest radiographs $(A-C)$ with corresponding chest computed tomography $(E-G)$ over a 10-year period showing progressive enlargement of the rightsided aneurysmal proximal pulmonary artery in a 40-year-old man with congenital PAH related to a ventricular septal defect and double outlet right ventricle. Despite maximising PAH therapies, the patient presented with ultimately fatal pulmonary haemorrhage (D). (H) CT coronary angiogram (CTCA) in a 20-year-old woman presenting with intense exertional chest pain. She had a background of Eisenmenger syndrome due to a large uncorrected ventricular septal defect. Reconstruction CT images show enlargement of the aneurysmal main pulmonary artery (MPA) $(6 \times 7 \mathrm{~cm}$, since her previous CT in 2013), with apparent distortion and severe compression of the left main stem and proximal left anterior descending coronary arteries (LCAs), as shown in the figure. The patient proceeded to coronary angiography via a femoral approach, which identified a sub-occlusive compression of the LAD 1-2 mm from the origin. An $18 \times 4.0 \mathrm{~mm}$ Everolimus eluting Xience stent was inserted. She was monitored closely on intensive care post procedure and had immediate relief of symptoms. She received Aspirin and Clopidogrel for 12 months. 
Exercise testing for detection of coronary artery disease is often not feasible as patients with PAH may be unable to exercise or cannot reach target heart rate, and the interpretation of ECGs is made difficult by baseline changes. Nuclear myocardial imaging can detect ischaemia of both left and right ventricular myocardium. ${ }^{13}$ Invasive coronary angiography should be used in patients at high risk of ischaemic heart disease, in whom non-invasive investigations were either positive or failed to exclude coronary artery disease. CT coronary angiography (CTCA) may also be used to non-invasively delineate coronary anatomy for intrinsic disease and can be a useful first-line test in cases of suspected extrinsic left coronary compression by a dilated proximal pulmonary artery (figure $1 \mathrm{H}$ ). In such cases, intravascular ultrasound can also be used to detect dynamic reduction in coronary cross-sectional area. ${ }^{14}$
Care should be taken before administering anti-platelets or anticoagulants to patients who have a history of haemoptysis or other significant bleeding (especially patients with Eisenmenger syndrome), unless there is high clinical suspicion of ongoing ischaemic heart disease or confirmed severe coronary atherosclerosis. Nitrates should generally be avoided in patients with $\mathrm{PAH}$, especially those with cyanotic CHD or receiving a phosphodiesterase (PDE) -5 inhibitor or guanylate cyclase (GC) stimulator, due to the risk of increasing cyanosis and profound hypotension, respectively. $\beta$ blockers are also generally contraindicated in patients with PAH with RV dysfunction ${ }^{15}$ (see arrhythmia section). Percutaneous coronary stenting, either for significant atherosclerotic coronary artery disease, or of extrinsic coronary compression, appears relatively safe and effective, 791014 but should be undertaken in centres with expertise in coronary

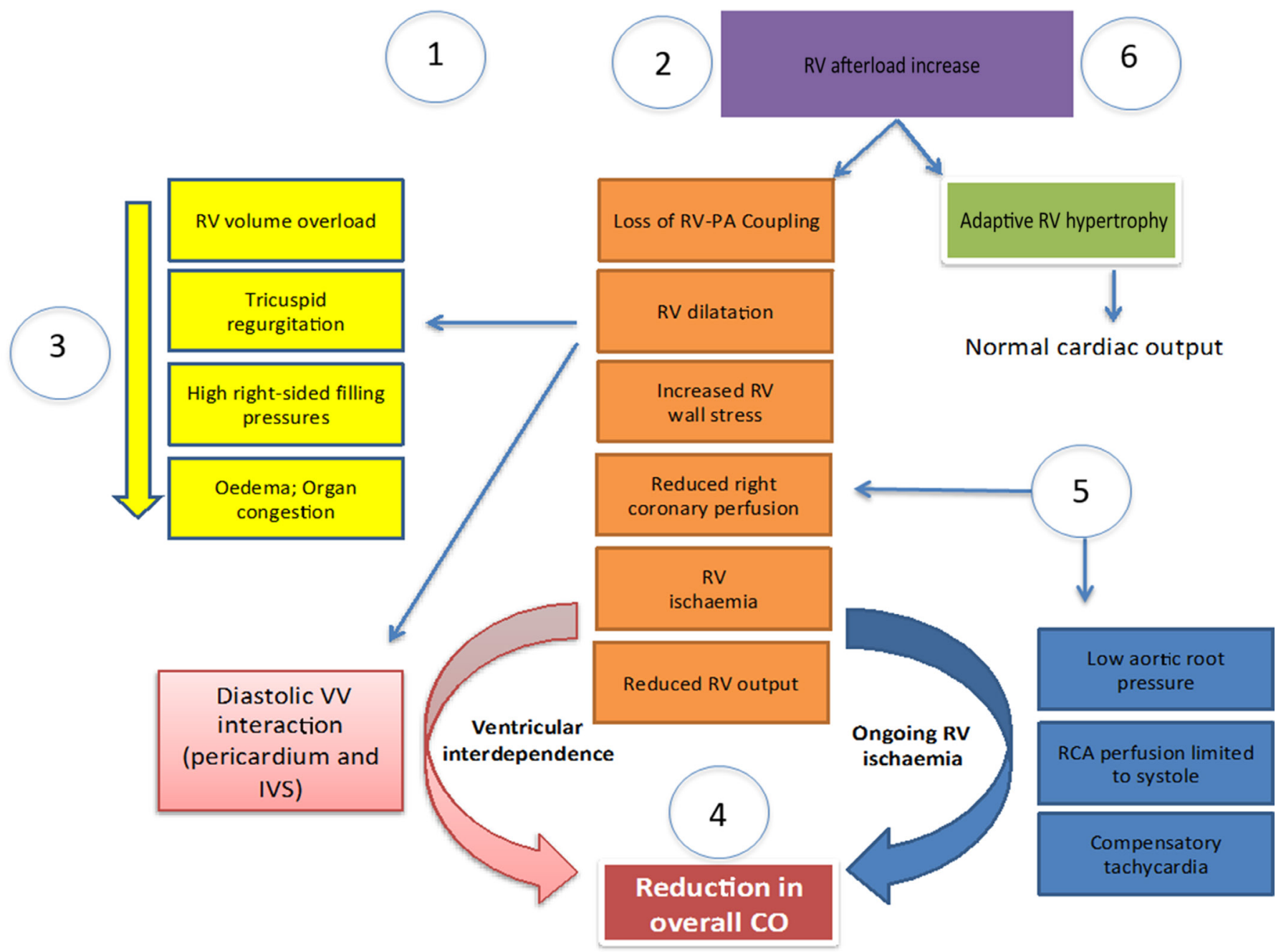

Figure 2 Mechanisms of right ventricular failure after pulmonary hypertensive crisis in patients with pulmonary arterial hypertension (PAH). A pulmonary hypertensive (PH) crisis is a physiological state in the setting of severe PH (usually PAH), in which a sudden increase in right ventricular (RV) afterload (purple box, top) leads to severe RV dysfunction, an acute fall in cardiac output and systemic hypotension. The resulting precipitous fall in aortic root and right coronary perfusion pressure causes further RV ischaemia (right hand, blue boxes), and a downward spiral of RV failure that is extremely difficult to interrupt. Compounded by this is the onset of diastolic left ventricular (LV) failure due to diastolic ventriculo-ventricular (VV) interactions (left hand, pink box), with further tricuspid regurgitation (yellow boxes). Black circles 1-6 correspond to the management steps 1-6 within the text, with (1) identification and treatment of precipitating factors; (2) maintenance of oxygen saturations, (3) optimising fluid balance, usually by reduction of preload using intravenous diuretics and spironolactone; (4) optimising cardiac output (CO) and myocardial contractility with inotropes; (5) maintaining systemic blood pressure, aortic root pressure and right coronary arterial (RCA) perfusion pressure (using vasopressors such as norepinephrine, if needed); (6) reducing pulmonary vascular resistance using PAH-targeted therapies. Steps 4-6 are often initiated concurrently. IVS, interventricular septum. 
Box 2 Suggested inpatient investigations in a patient with known PAH presenting with RV failure

Clinical features: note nature of symptom progression, exercise intolerance, worsening oedema, change in syncope/presyncope, chest pain, previous PAH therapy, cessation or poor compliance of PAH therapy, quality of life. Note change in WHO FC and 6MWD.

Examination findings: signs of RV failure: weight gain, tachycardia, low BP, raised JVP, oedema (leg, ascites, pulsatile hepatomegaly, pericardial/pleural effusions). Assess for signs of IE, murmurs, new CTD features, DVT, any reversible process.

Assessment of RV function: ECG onset of RV strain, rising BNP, echocardiography, CT cardiac chamber dimensions, CMR, pulmonary haemodynamics at RHC.

Echocardiographic signs*: large right atrial area, presence of pericardial effusiont, RV dilatation/dysfunction.

RHC: RAP $>12-14 \mathrm{mmHg}$, low cardiac output, low $\mathrm{SvO}_{2}$.

\section{Investigations to exclude reversible causes}

Electrolyte or metabolic imbalance: biochemistry, liver, thyroid function.

Infection: CRP, septic screen (blood, urine, sputum), ABG,

lactate, imaging.

Arrhythmia: 12 lead ECG, telemetry.

Pulmonary embolism: CTPA, consider $d$ dimer.

Remember distal CTEPH (VQ scan if available/patientstable)

Exclude thrombophilia

Myocardial ischaemia: Troponin assay, may need CTCA, coronary angiography+IVUS

Exacerbation of underlying lung disease: $C X R, A B G$, peak flow chart, consider HRCT.

Onset of sleep-disordered breathing: Embletta, $\mathrm{O}_{2} / \mathrm{CO}_{2}$ study

CTD-PAH: exclude lupus flare and antiphospholipid syndrome.

\begin{abstract}
*PAP may not be a good marker of disease severity when RV dysfunction is established. Low PA pressures may indicate a failing $\mathrm{RV}$ rather than improved PH. More important are size of RA and RV, presence of pericardial fluid, and function of the RV.

tDo not routinely tap pericardial effusions, which are usually a sign of decompensated RV failure and rarely cause tamponade in patients with a hypertensive RV.

6MWD, 6 min walking distance; $A B G$, arterial blood gas; CMR, cardiac magnetic resonance; CRP, C reactive protein; CTCA, CT coronary angiogram; CTD, connective tissue disease; $\mathrm{CTEPH}$, chronic thromboembolic PH; CTPA, CT pulmonary angiogram; CXR, chest radiography DVT, deep venous thrombosis; HRCT, high-resolution $\mathrm{CT}_{\text {; }}$ IE, infective endocarditis; IVUS, intravascular ultrasound; JVP, jugular venous pressure; $\mathrm{PAH}$, pulmonary arterial hypertension; RAP, right atrial pressure; $\mathrm{RHC}$, right heart catheterisation; $\mathrm{RV}$, right ventricular; $\mathrm{SvO}_{2}$, mixed venous oxygen saturation; $\mathrm{VQ}$, ventilation perfusion; WHO FC, WHO functional class.
\end{abstract}

intervention and $\mathrm{PAH}$, with expert anaesthetic backup to manage potential complications, avoiding sedation or general anaesthesia whenever possible.

\section{Suggested approach}

Chest pain in PAH may result from 'usual common causes' as well as PAH-specific causes. Standard initial investigations should always be performed and interpreted in the context of the clinical presentation and comorbidities, followed by PAH-specific investigations, in close liaison with the PH specialist centre. Extrinsic left main stem compression should be excluded in patients with $\mathrm{PAH}$ with recurrent and/or anginal pain, with patients urgently transferred to the $\mathrm{PH}$ centre for further assessment including CTCA, interventional cardiology support and augmentation of $\mathrm{PH}$ therapies as appropriate.

\section{Management of RV failure in a patient with PAH}

RV failure is a major determinant of morbidity and mortality in $\mathrm{PAH},{ }^{16}$ and worsening $\mathrm{RV}$ function in PAH may result from gradual disease progression or from an acute insult, including all 'CRASH scenarios' such as arrhythmia, bleeding, infection (including sepsis), systemic hypotension or pulmonary embolism (PE). Managing the primary cause of deterioration is needed, for example in PE where management principles are usually similar, while considering protection of the RV. It is worthy of mention that the usual indication for thrombolysis in a patient with PAH and pre-existing RV dysfunction would differ to a patient with acute RV dysfunction and a naive RV. In patients with PAH the effect of any of these insults on the RV can be unpredictable; patients may require escalation of $\mathrm{PAH}$ therapy or mechanical support, and emergency transfer to a $\mathrm{PH}$ centre is advisable. Any rise in PVR triggers further RV dysfunction ${ }^{17}$ and impaired RV adaptation to rapid increases in afterload ${ }^{18}$ results in 'ventriculo-arterial uncoupling', RV distension, myocardial oxygen consumption/delivery imbalance and heart failure. ${ }^{19}$ Heart failure in PAH is rarely just a right-sided phenomenon. Significant RV-left ventricular (LV) interdependence ${ }^{20}$ exists in the setting of RV pressure and/or volume overload. This leads to impaired LV filling contributing to the overall fall in cardiac output and heart failure (figure 2).

\section{Critical care management of RV failure in PAH}

Patients with decompensated PAH and RV failure should be moved to a critical care environment. Appropriate investigations (box 2) and monitoring (table 1) aim to identify and reverse precipitating factors, ${ }^{21}$ especially those associated with high mortality characteristics (box 3). A rising venous lactate and low central venous saturations $\left(\mathrm{ScvO}_{2}\right)$ are simple markers of low cardiac output and are often under-utilised. Optimal fluid balance is paramount, as both hypovolaemia and hypervolaemia may be detrimental to cardiac output (see table 1). In patients with hypervolaemia (the most common situation), reducing RV preload through diuresis (often using a continuous intravenous furosemide infusion) will improve RV function, RV-LV interdependence and LV diastolic compliance. ${ }^{19}$ Patients with decompensated RV failure develop secondary hyperaldosteronism and addition of an aldosterone antagonist, such as spironolactone, to a loop diuretic can be useful. ${ }^{22}$

All attempts should be made to reduce PVR in patients with $\mathrm{PAH}$ presenting with heart failure. Correction of factors that can cause a rise in PVR, such as alveolar hypoxia, hypercapnia and acidaemia, is needed, with the proviso that intubation and positive pressure ventilation should be avoided whenever possible due to detrimental effects on PVR and RV function. In severely decompensated patients already on oral pulmonary vasodilators (such as a phosphodiesterase inhibitor or endothelin receptor antagonist), intravenous prostacyclin should be initiated. ${ }^{19} 2324$ Nebulised prostacyclins may be useful in the setting of decompensated RV failure, but intravenous prostacyclin remains the most efficacious of all therapies in this setting. Intravenous prostacyclin is up-titrated through a tunnelled or central line 
Table 1 Levels of suggested monitoring in a patient with PAH and RV dysfunction. The ward and level of monitoring will depend on the adverse prognostic factors as listed in box 3, with high-risk patients requiring advanced level monitoring at least in a level two ward or high dependency or intensive care unit

\begin{tabular}{ll}
\hline Monitoring RV failure in a patient with PAH & Target aims \\
\hline Clinical signs: & \\
\hline WHO functional class & Improvement \\
ECG & Sinus rhythm, less tachycardia \\
Monitor daily weight, fluid balance, JVP, and signs of overload & Negative fluid balance, JVP falling \\
BP & Target BP $>90 \mathrm{mmHg}, \mathrm{MAP}>70 \mathrm{mmHg}^{*}$ \\
Renal and liver function & Improving renal, liver function \\
BNP & Fall in BNP during the hospital admission (if rechecked)
\end{tabular}

Baseline haemodynamic monitoring: on level 1 ward

Telemetry, non-invasive BP (as above), lactate

$\mathrm{SaO}_{2}$

Echocardiographic signs: right atrial area, pericardial effusion, RV function

Urine output (catheterise)

Consider RHC (see below)

Lactate $<2 \mathrm{mmol} / \mathrm{L}$

$\mathrm{SaO}_{2}>90 \%$

Improved RV parameters (and PAP)

Increased urine output. Urine output aim for $>0.5 \mathrm{~mL} / \mathrm{kg} /$ hour

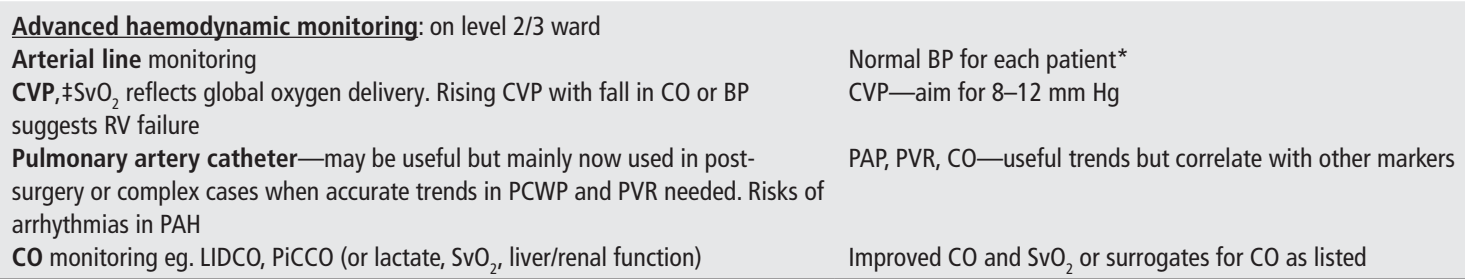

with invasive haemodynamic monitoring using central venous pressure (CVP), arterial line, and vasopressor and/or inotropic support, as appropriate. Pulmonary artery (Swan Ganz) catheter monitoring is now rarely used to measure cardiac output, although regular assessment of PVR may be helpful in selective patients with treatment-refractory RV failure and in postoperative cases. Other less invasive methods to measure cardiac output include pulse index continuous cardiac output (PiCCO), lithium dilution techniques (LIDCO) or similar devices. In practice, most patients have invasive CVP (right atrial pressure) measurement, serial echocardiography and surrogates for cardiac output, such as serial lactate or $\mathrm{SvO}_{2}$ measurements, renal and hepatic function for monitoring purposes. When initiating intravenous prostanoid therapy, haemodynamic support may be required, especially in patients with low cardiac output: patients in acute renal injury may respond well to coadministration of an inotrope, such as dobutamine or dopamine. Other patients may also require a vasopressor. See below and table 2 for emergency drugs used in RV failure including pulmonary vasodilators and other vasoactive agents.

\section{Vasopressors and inotropes in RV failure}

Prompt maintenance of aortic root pressure, and thus right coronary perfusion pressure, is critical to prevent $\mathrm{RV}$ ischaemia. This can be achieved by maintaining systemic blood pressure using a systemic vasopressor agent, while also reducing RV preload and afterload hence minimising RV wall stress. Of the systemic vasopressors, norepinephrine is used as a first-line agent, with vasopressin often used as a second-line agent. Norepinephrine may cause pulmonary vasoconstriction at very high concentrations, but rarely at doses up to $0.5 \mu \mathrm{g} / \mathrm{kg} / \mathrm{min} .{ }^{25}$ Low-dose vasopressin, acting via non-adrenergic V1 receptors, may have a better pulmonary to systemic vascular resistance (SVR) profile, with theoretical benefit in PH as PVR is reduced while SVR augmented; however limited clinical data support its use in $\mathrm{PAH}^{26-28}$ (table 1).

Patients with PAH, RV failure and low cardiac output may develop end-organ dysfunction, such as acute kidney injury, requiring inotropic support. Dobutamine, a $\beta 1$ and 2 agonist, is most commonly used, and is the drug of choice. Dopamine is an alternative, used in renal dysfunction due to its additional natriuretic properties. ${ }^{19} 2324$ All catecholamines may induce a dose-related sinus tachycardia. ${ }^{29}$ Inodilating agents, including levosimendan (see below) and the phosphodiesterase type 3 (PDE3) inhibitor, milrinone, are attractive and increasingly used in RV failure in critical care settings. ${ }^{24}$ Through PDE3 inhibition, both agents augment myocardial function and reduce PVR. Levosimendan also increases the sensitivity of cardiac myofilaments to calcium ions, thereby improving contractility without increasing myocardial oxygen demand. Levosimendan causes systemic and pulmonary vasodilatation by stimulating potassium channels and PDE3 inhibition. Both agents improve cardiac output and pulmonary haemodynamics in models of RV dysfunction, for example following acute pulmonary embolus, ${ }^{30}$ and in patients with group $2 \mathrm{PH}$ following cardiac surgery or transplantation. Similar improvements in RV function in PAH are however currently limited to case reports for levosimendan, for example in CHD-PAH ${ }^{31}$ and CTEPH. ${ }^{32}$ Several experimental models support the use of intravenous milrinone in acute RV failure with $\mathrm{PH}$, and clinical studies and experience suggest this agent is well tolerated in RV dysfunction with $\mathrm{PH}$, for example following cardiac surgery, ${ }^{33}$ during liver transplantation surgery with portopulmonary $\mathrm{PH}^{34}$ also used in nebulised form in a $\mathrm{PH}$ crisis in a patient with idiopathic PAH. ${ }^{35}$ Both milrinone and 
Table 2 Drugs available in the UK to treat acute PH to avoid PH crisis with RV failure

\begin{tabular}{|c|c|c|}
\hline Pulmonary vasodilators to reduce RV afterload & Vasopressors to maintain right coronary perfusion* & Inotropes to increase myocardial contractility \\
\hline Inhaled agents & Sympathomimetic agents & Catecholamines: \\
\hline Inhaled iloprost $2.5-5 \mu \mathrm{g}, 6-9 /$ day & Norepinephrine $0.01-0.5 \mu \mathrm{g} / \mathrm{kg} / \mathrm{min}$ & Dobutamine $(2.5-10 \mu \mathrm{g} / \mathrm{kg} / \mathrm{min}) \S$ \\
\hline Continuous inhaled or nebulised agents: & (in animal models no increase in PVR in this range) & Dopamine $5-10 \mu \mathrm{g} / \mathrm{kg} / \mathrm{min} \S$ ** \\
\hline Nebulised epoprostenol $30-40 \mathrm{ng} / \mathrm{kg} / \mathrm{min}$, over $10-20 \mathrm{~min}$ & Non-sympathomimetic vasopressors & Inotropic via $\beta 1$ agonism \\
\hline Inhaled milrinone $1 \mu \mathrm{g} / \mathrm{kg} / \mathrm{min}$, aerosolised over $10-20 \mathrm{~min}$ & Vasopressin & Vasodilatation (dobutamine if $>10 \mathrm{mcg} / \mathrm{kg} / \mathrm{min}$ ) \\
\hline Inhaled nitric oxide $5-40 \mathrm{ppm}$ & (Argipressin, 20 units $/ \mathrm{mL}$ ) & Tachycardia (dose dependent) \\
\hline Intravenous agents & 'Low dose' infusion $0.01-0.04$ units/min. Adverse & Inodilators: \\
\hline Intravenous epoprostenol, starting at $1-2 \mathrm{ng} / \mathrm{kg} / \mathrm{min}$, & effects $>0.06$ units $/ \mathrm{min}$ & Milrinone $(50 \mu \mathrm{g} / \mathrm{kg}$ over $10 \mathrm{~min}$ then $0.375-0.75 \mu \mathrm{g} / \mathrm{kg} /$ \\
\hline increasing by $1-2 \mathrm{ng} / \mathrm{day}$ up to $10 \mathrm{ng} / \mathrm{kg} / \mathrm{min}$ or higher & Bolus (in PH crisis) 10 units & $\min$ infusion) \\
\hline Intravenous iloprost $1-5 \mathrm{ng} / \mathrm{kg} / \mathrm{min}$ & Terlipressin $\ddagger$ & PDE3 inhibitor, increases CAMP \\
\hline \multirow{6}{*}{$\begin{array}{l}\text { Sildenafil ( } 0.05-0.43 \mathrm{mg} / \mathrm{kg} \text {, usually } 10-20 \mathrm{mg} \text { bolus). } \\
\text { Intravenous equivalent dose is } 50 \% \text { of oral dose. Infusion } \\
\text { (Revatio, } 10 \mathrm{mg} \text { in } 12.5 \mathrm{~mL} \text { vial) starts at } 1.25 \mathrm{mg} / \mathrm{hour} \\
\text { (max effect in } 20 \mathrm{~min} \text { ) }\end{array}$} & $\begin{array}{l}\text { Long half life so intermittent bolus, } 0.5 \mathrm{mg} \text { to total } 2 \mathrm{mg} \\
\text { Suggested vasopressor protocol for resistant hypotension }\end{array}$ & $\begin{array}{l}\text { Can cause systemic vasodilatation and arrhythmias. Use } \\
\text { with a vasopressor }\end{array}$ \\
\hline & with $R V$ dysfunction in $P H$ & Levosimendan \\
\hline & First line: Norepinephrine, up to $0.1 \mu \mathrm{g} / \mathrm{kg} / \mathrm{min}$, then & Calcium sensitiser and PDE3 inhibitor \\
\hline & consider additi & Initial infusion 0.05 then $0.1 \mu \mathrm{g} / \mathrm{kg} / \mathrm{min}$ (without a bolus) \\
\hline & & $\mathrm{Ca}$ \\
\hline & & May also require vasopressor to manage hypotension \\
\hline
\end{tabular}

\footnotetext{
${ }^{*}$ Central venous access with invasive arterial line pressure monitoring required. Consider also CVP, cardiac output and/or PA pressure monitoring.

tUp-titrated according to tolerable side effects (notably headache, flushing, nausea, vomiting, diarrhoea, jaw pain, muscle aches).

‡Unlicensed. Only use if vasopressin not available.

§Up to $5 \mu \mathrm{g} / \mathrm{kg} / \mathrm{min}$ can be administered peripherally, above this should have central venous access. Do not use above $10 \mu \mathrm{g} / \mathrm{kg} / \mathrm{min}$.

**D1 agonism 'renal' dopamine effect, may be helpful in low GFR state.

CVP, central venous pressure; GFR, glomerular filtration rate; PDE, phosphodiesterase; $\mathrm{PH}$, pulmonary hypertension; PVR, pulmonary vascular resistance; RV, right ventricular.
}

levosimendan can cause atrial arrhythmias (up to $20 \%$ of cases) and systemic hypotension, hence should be used with caution. In patients with end-stage RV failure not responding to conventional therapy, extracorporeal life support may be considered in cardiothoracic and transplant centres to support the failing RV as a bridge to lung transplantation, which may be life saving in eligible patients.

\section{Suggested approach}

The approach to patients with PAH presenting with RV failure should be systematic and is illustrated in conjunction with this approach in figure 2: (1) identify and treat precipitating factors; (2) maintain oxygen saturations $\left(\mathrm{SaO}_{2}>90 \%\right.$ or close to previous levels in chronically cyanotic individuals); (3) optimise fluid balance, usually by reduction of preload using intravenous diuretics and spironolactone, aiming for 1-1.5 litres negative balance per day (more aggressive negative balance can be detrimental to RV function); (4) optimise cardiac output and myocardial contractility with inotropes; (5) maintain systemic blood pressure, aortic root pressure and RCA perfusion pressure (using vasopressors such as norepinephrine, if needed); (6) reduce PVR using PAH-targeted therapies. Steps 4-6 are often initiated concurrently.

\section{Management of arrhythmias in a patient with $\mathrm{PAH}$}

Arrhythmias are frequent in PAH and often cause clinical deterioration requiring hospitalisation, or may result in sudden death. ${ }^{36}{ }^{37}$ Atrial flutter and fibrillation are the most common supraventricular tachycardias (SVTs) in PAH, with an incidence of $2.8 \%$ per annum. ${ }^{36}$ In advanced PAH, increased atrial stretch and myocardial fibrosis predisposes patients to SVTs, with a 5 -year cumulative frequency of $25.1 \% .^{38-40}$ SVTs are especially common in CHD-PAH and are often incisional, that is result from previous surgical scars, or right atrial dilatation, that is raised RV diastolic pressure from valve dysfunction or intra-cardiac shunts. ${ }^{412}$ Increased sympathetic activity in idiopathic PAH may also predispose patients to arrhythmias, ${ }^{43}$ and as in any
Box 3 Parameters identifying 'high-risk' patients with RV failure.

Certain clinical indicators suggest increased mortality in patients with PAH. This list includes characteristics that would suggest a poor prognosis on hospital admission. ERS/ESC 2015 guidelines suggest that high-risk patients have a 1 -year mortality of $10 \%,{ }^{3}$ and although few studies are available for patients with PAH requiring admission to HDU (level 2) or ICU, mortality is likely to be at least $40 \%$ and associated with the factors listed below. ${ }^{21}$

\section{High-risk patients with PAH \\ Clinical features:}

- Signs of RV failure (peripheral oedema, raised JVP, hepatomegaly, ascites)

- Rapid progression of symptoms

- Syncope

- WHO FC IV at presentation

Exercise parameters:

- Last $6 \mathrm{MWD}<165 \mathrm{~m}$

- CPET low VO $\max <11 \mathrm{ml} / \mathrm{min} / \mathrm{kg}$, high VE $/ \mathrm{VCO}_{2} \geq 45$

Haemodynamics:

- Right atrial area $>26 \mathrm{~cm}^{2}$

- Pericardial effusion

- RAP> $>14 \mathrm{mmHg}$

- $\mathrm{Cl}<2 \mathrm{~L} / \mathrm{min} / \mathrm{m}^{2}$

- $\mathrm{SvO}_{2}<60 \%$

Features on hospital admission: low systemic blood pressure, hyponatraemia, raised CRP/sepsis, raised creatinine, $\mathrm{BNP}>300 \mathrm{ng} / \mathrm{l}$ (and rising).

6MWD, 6 min walking distance; BNP, brain natriuretic peptide; $\mathrm{Cl}$, cardiac index; $C P E T$, cardiopulmonary exercise testing; $C R P, C$ reactive protein; $\mathrm{RAP}$, right atrial pressure; $\mathrm{RV}$, right ventricular; $\mathrm{SvO}_{2}$, mixed venous oxygen saturations; WHO FC, WHO functional class. 
setting, thyroid dysfunction and electrolyte disturbances should be excluded.

There is little evidence on the optimum management of arrhythmias in PAH. The outcome of patients with $\mathrm{PAH}$ presenting with SVT relates to the type of arrhythmia and on prospects for restoration of sinus rhythm. ${ }^{36}$ With haemodynamic compromise, arrhythmias should be treated promptly, usually through direct current cardioversion (DCCV) with careful post-procedure monitoring until the low cardiac output state has recovered. In SVTs involving the AV node, adenosine may be considered prior to DCCV. ${ }^{36}$ DCCV requires sedation or general anaesthesia, both of which carry significant risks in PAH. Senior cardiac anaesthetic input is therefore required. Regardless, cardioversion of a patient with significant $\mathrm{PAH}$ and a fast arrhythmia should be prompt to avoid progressive, potentially irreversible haemodynamic decompensation. While amiodarone may be effective in cardioverting SVTs, its relatively slow onset of action makes it less attractive in the acute setting. Amiodarone is useful for maintenance of sinus rhythm following DCCV for atrial fibrillation, which has a high recurrence rate, or other recurring arrhythmias that cannot be ablated. Anticoagulation should be considered for stroke prevention; the CHADS2 (congestive heart failure, hypertension, age $>75$ years, diabetes, stroke or PE) VASc score used to calculate stroke risk in atrial fibrillation for the general population ${ }^{44}$ may be used in PAH but has not been validated in this population.

In all cases, a tertiary $\mathrm{PH}$ centre should be contacted for advice for any patient with PAH presenting with an arrhythmia. Patients who are haemodynamically stable should be transferred, especially when the time of arrhythmia onset is unclear or exceeds 24-48 hours. In the latter, DCCV under transoesophageal echocardiographic guidance to exclude intra-atrial thrombus is best performed in specialist centres with anaesthetic and imaging expertise. Elective ablation of atrial flutter, once stabilised, is often safe and effective in $\mathrm{PAH}$, may reduce recurrence rate and thus requirement for DCCV and general anaesthesia. ${ }^{45}$

$\beta$ blockers should be used with caution in $\mathrm{PAH}$, starting with small doses and up-titrating slowly, due to their negative inotropic effect which may be poorly tolerated in patients with poor RV function. ${ }^{46}$ Calcium channel blockers are also best avoided for the same reason and their effect on systemic vasodilatation which can be detrimental in this population. Clear recommendations on the use of antiarrhythmics cannot be made in view of the lack of evidence, and preference should be given to medication with little or no negative inotropic effect (eg, amiodarone), although long-term use can cause serious thyroid and lung complications. ${ }^{47}$ Catheter ablation should be considered for cases of recurrent arrhythmia, especially when refractory to medical treatment. ${ }^{45} 48$

Arrhythmia appears to relate to mid-term outcome in PAH. ${ }^{36-38}$ It is unlikely that arrhythmia alone leads to worse prognosis, but instead represents a surrogate of advanced PAH, with deteriorating RV function and tricuspid regurgitation. ${ }^{38}$ Restoration of sinus rhythm should always be pursued, and may be related to better prognosis in PAH. Malignant tachycardias are rare in $\mathrm{PAH}$, with $8 \%$ of 132 witnessed cardiac arrests relating to ventricular fibrillation, ${ }^{49}$ and are reported in patients with scleroderma with myocardial fibrosis. ${ }^{50}$ Sudden death does, however, occur in $\mathrm{PAH}$ and all patients with $\mathrm{PAH}$ presenting with sustained malignant ventricular tachycardia (VT) or ventricular fibrillation should be investigated for extrinsic compression of the left anterior descending coronary artery by a dilated hypertensive pulmonary artery (see Chest Pain section). Patients with idiopathic $\mathrm{PAH}$ and autonomic dysfunction are thought to be at higher risk of ventricular arrhythmias. ${ }^{43}$ Patients with congenital heart disease (CHD)-related PAH may also be prone to malignant tachycardias relating to the cardiac defect itself as well as surgical scars, with non-sustained VT in 21\% and VF in $3 \%$ at 4 years of follow-up. ${ }^{51}$ Surrogates of VT such as QTc and QT dispersion are described in PAH and an increased frequency of ventricular and supraventricular ectopics described in Eisenmenger syndrome. ${ }^{52}$ How these relate to the risk of malignant arrhythmia in PAH remains unknown.

\section{Suggested approach}

Rapid restoration of sinus rhythm is key for atrial arrhythmias in PAH, usually requiring electrical DCCV with senior anaesthetic support. Precipitants such as biochemical disturbance or myocardial ischaemia should be corrected. Anticoagulation should be considered based on risk/benefit assessment (such as the CHADS2-VASc score in atrial fibrillation). Avoid $\beta$ blockers and calcium channel blockers in patients with severe RV dysfunction. Catheter ablation may be considered in the acute setting in tertiary centres in patients with stable disease under close monitoring and in those with recurrent arrhythmias.

\section{Management of sepsis in PAH}

Infection and sepsis are associated with significant morbidity and mortality in PAH. ${ }^{1}$ The triggering of vasoactive and microthrombotic inflammatory processes causes direct and indirect injury to the pulmonary circulation (with subsequent increase in PVR), compounded by sepsis-induced myocardial depression and systemic vasodilation. ${ }^{3}$ In patients with RV failure, bacterial translocation from the bowel (including occasional spontaneous bacterial peritonitis) may be a major source of sepsis, and have potentially challenging therapeutic consequences if surgery is required. Sepsis is the most common reason for patients with $\mathrm{PAH}$ to be admitted to critical care. ${ }^{21}$ General management principles (according to the international Surviving Sepsis Guidelines) apply, ${ }^{4}$ including early antibiotic administration and a goal-directed approach, although rapid volume resuscitation is clearly potentially hazardous, with close haemodynamic monitoring important (box 2). Management principles described in the Management of RV failure in PAH section should be followed. If required, respiratory support using continuous positive airways pressure (CPAP) and non-invasive ventilation (NIV) are generally well tolerated, although in-hospital mortality remains high in patients with PAH (12\% with NIV in a recent US-wide survey). Nasal high-flow oxygen (Optiflow), which provides low $\left(2-3 \mathrm{cmH}_{2} \mathrm{O}\right)$ levels of positive pressure and high $\mathrm{FiO}_{2}$, is particularly well tolerated in patients with $\mathrm{PAH} .{ }^{53}$ Decisions on intubation and invasive mechanical ventilation will depend on the aetiology and potential for reversibility. Endotracheal intubation is high risk in PAH due to haemodynamic collapse relating to systemic vasodilating effects of anaesthetic induction agents. Positive pressure ventilation and positive end-expiratory pressure increases alveolar plateau pressure and PVR and diminishes venous return, potentially exacerbating RV failure. ${ }^{6}$ A recent large analysis of patients with PAH showed a mortality of $39 \%$ in patients subjected to invasive positive pressure ventilation. ${ }^{53}$ Early referral of appropriate patients with PAH to a critical care environment for close haemodynamic monitoring is, therefore, strongly advised in those needing cardiorespiratory support.

\section{Management of infections in PAH}

Infections are poorly tolerated in patients with $\mathrm{PAH}$, and should be excluded in patients with clinical deterioration. Chest 
radiograph, urinalysis and blood cultures should form part of the routine assessment of unwell patients with $\mathrm{PAH}$.

\section{Indwelling line sepsis}

Infection of tunnelled central venous access (eg, Groshong or Hickman lines) in patients using parenteral prostanoids is a serious complication with potentially serious consequences. ${ }^{9}$ Line infection should be differentiated from tunnel infection (tunnelitis). Both may occur through non-adherence to strict hygiene measures with line infection frequently resulting in sepsis, although often may happen in the most fastidious of patients. Line sepsis can occur in the absence of features of sepsis and fever, although a normal C-reactive protein is unusual. Diagnosis of a catheter-related blood stream infection requires confirmation of positive cultures from a peripheral blood culture, matched with either the culture of the catheter introducer (more sensitive than the catheter tip in this setting), and/or a blood culture taken from the line itself. ${ }^{54}$ Line removal is indicated in confirmed line infection and often in tunnelitis. ${ }^{55}$ Prior to removal of the line or taking cultures from the potentially infected line, the prostacyclin infusion should be established via an alternative route, ideally a peripherally inserted central line or central line, as temporary cessation of the infusion may precipitate a $\mathrm{PH}$ crisis due to the short half-life of prostacyclin. When line infection is likely, early initiation of broad-spectrum antibiotics, following local microbiology advice (eg, teicoplanin and tazocin or vancomycin if MRSA suspected) while awaiting cultures is a reasonable approach, with an important decision being the removal of the potentially infected line. Replacement of the tunnelled line should await full recovery.

\section{Infective endocarditis (IE)}

IE is a recognised complication in patients with CHD-PAH, particularly patients with intra-cardiac shunts, valve disease and previous surgery. ${ }^{785}$ Poor dentition and dental procedures are common precipitants. ${ }^{56}$ Moreover, patients with PAH with indwelling catheters are at risk of developing endocarditis as a result of a line infection, although in reality, this is not very common. Direct cardiac damage and septic emboli may occur as a result of IE, leading to systemic abscess formation, the most feared of which are cerebral abscesses (to be suspected in all patients with cyanosis and new neurological signs and symptoms, see below). Early intravenous antibiotic therapy is essential to minimise complications. National and international guidelines provide specific recommendations for the prophylaxis, diagnosis and treatment of IE. ${ }^{56}$

\section{Cerebral abscesses}

Septic emboli, especially in those with intracardiac shunts, lead to cerebral involvement, and can have serious sequelae. ${ }^{8}$ New neurological symptoms and deficits should be investigated immediately, especially in patients with cyanosis, with non-invasive imaging and lumbar puncture reserved for suspected meningoencephalitis. Urgent neurology/neurosurgical review is recommended with transfer to critical care for closer monitoring and sepsis management. Indwelling peripheral cannulae should be reviewed daily and removed if there is suggestion of infection.

\section{Suggested approach}

Early recognition, prompt targeted antibiotic treatment and close monitoring with appropriate critical care support are essential in the management of sepsis in patients with PAH. Removal of a tunnelled line is indicated in confirmed line infection. National and international guidelines provide specific recommendations for the management of infective endocarditis. For patients with confirmed cerebral abscess, urgent neurology/neurosurgical review is recommended.

\section{Management of haemoptysis in a patient with $\mathrm{PAH}$}

Spontaneous haemoptysis is not uncommon in Eisenmenger syndrome but can also occur in other types of $\mathrm{PH}$, such as CTEPH. ${ }^{41-257-62}$ In a large registry of patients with CHD-PAH, haemoptysis was the presenting symptom in $7 \%$ of cases. ${ }^{62}$ Haemoptysis also appears to be more common in patients with heritable PAH who are BMPR2 mutation carriers. ${ }^{63}$ It can be mild or severe and is often recurrent. It is usually self-limiting, but may become life threatening. Mechanisms for haemoptysis are thought to be a combination of high pulmonary artery pressure, bronchial artery hypertrophy, neovascularisation and anticoagulation ${ }^{64}$ but also pulmonary embolism, particularly in patients with cyanosis. ${ }^{60}$ Rarely, massive haemoptysis may follow rupture of proximal PA aneurysms ${ }^{65} 66$ (figure $1 \mathrm{~A}-\mathrm{G}$ ).

Prognosis and management in haemoptysis depends on the PAH subtype, the aetiology and severity of the haemoptysis. ${ }^{66}$ In a single centre study of 21 cases, mortality was high in patients without CHD-PAH (31\% 28-day mortality and 54\% 1-year mortality), whereas no deaths were observed in patients with CHD-PAH. ${ }^{61}$ Massive haemoptysis carries a mortality of up to $50 \%$ if untreated.

There is little evidence on the optimal management of haemoptysis in PAH. All haemoptysis should be considered an emergency until proven otherwise and an $\mathrm{ABC}$ protocol (airway/breathing/circulation) followed. The bleeding should be first localised on CT or chest radiograph to guide emergency management. Anaemia should be corrected, coexistent infection treated and supplemental oxygen is recommended until the bleeding stops. Anticoagulants should be withheld or reversed as appropriate, including prothrombin concentrate for vitamin $\mathrm{K}$ antagonists and the recent 'antidotes' for the direct oral anticoagulants. The use of tranexamic acid should be considered in large volume haemoptysis as a bridge to an interventional procedure (eg, bronchial arterial embolisation (BAE)), but long-term use is not recommended, particularly for minor bleeds, notably in patients with Eisenmenger syndrome in whom thrombotic/ embolic events can be devastating. ${ }^{6768}$ Recombinant factor VIIa (rFVIIa, Novoseven) may play a role when other treatments have failed and conventional clotting parameters have been corrected, ${ }^{69}$ although no reports are available in patients with $\mathrm{PH}$. Intravenous vasoconstrictors such as desmopressin can be considered in life-threatening haemoptysis, monitoring for further haemodynamic instability, water retention and sodium imbalance. ${ }^{6270}$

Bronchial collaterals are more notable in certain types of $\mathrm{PH}$ (in particular CTEPH; rare in idiopathic PAH), and BAE may be a means of controlling recurrent, persistent or massive haemoptysis. $^{71-74}$ Recurrent haemoptysis should certainly expedite transplant listing. ${ }^{75} \mathrm{BAE}$ is not routinely available and transfer to a regional centre where BAE is available is recommended. Recurrent bleeding occurs in over one-third of patients, ${ }^{606172}$ as new collateral vessels can develop, with interval repeat BAE procedures considered, especially in patients not for transplantation.

Flexible bronchoscopy should generally be avoided in patients with PAH due to hypoxia-induced increases in PVR, haemodynamic instability and bronchoscopy-associated bleeding. ${ }^{76} 77$ However, bronchoscopy may occasionally be helpful in the intubated patient with massive haemoptysis to locate the site of 
bleeding and guide bronchial artery embolisation ${ }^{78} 79$ or the use of blocking balloons (eg, Arndt blockers and tip Cohen blockers), which can stay in place by a three-way port after the scope is retracted while the patient is further stabilised.

\section{Suggested approach}

Management of small volume bleeds includes supplemental oxygen, cessation and/or reversal of anticoagulants and treatment of associated infection and PAH exacerbations. Therapeutic options for larger bleeds include tranexamic acid, activated factor VII, bronchial artery embolisation, the use of bronchial blockers (in the intubated patient) and transplantation.

\section{Surgery, anaesthesia and pregnancy in patients with PAH}

Surgery, general anaesthesia and pregnancy pose a particularly high risk to patients with PAH patients. Retrospective series of general surgical procedures suggest a 7\%-18\% mortality in PAH, with highest risk in major surgery, long procedures and emergency surgery. ${ }^{80-83}$ Overall, general anaesthesia is less well tolerated than regional anaesthetic techniques in $\mathrm{PAH}$, which include regional nerve blocks, epidural and combined (low-dose) spinal/ epidural techniques. Higher doses of spinal blockade lead to sympathetic blockade and a marked drop in preload, and are less well tolerated in patients with PAH. In addition, a drop in SVR worsens the right-to-left shunt in patients with Eisenmenger syndrome. Emergency procedures carry extremely high mortality in PAH. ${ }^{82}$ Preoperative RV dysfunction, poor exercise tolerance and higher WHO functional class, more complex and lengthy operations are associated with worse outcomes. ${ }^{80} 8283$ Aspects of general anaesthesia that pose a profound risk in a patient with PAH include anaesthetic induction agent-induced systemic vasodilatation, airway instrumentation causing tachycardia and the adverse effects of positive pressure ventilation; as well as adverse surgical effects (fluid shifts, head down positioning with $\mathrm{CO}_{2}$ insufflation for laparoscopic surgery, for example). If possible, PAH therapy should be maximised perioperatively; this may include switching to intravenous sildenafil to avoid missing doses while nil by mouth or converting to intravenous prostacyclin therapy. Careful planning is key, and mortality is reduced when procedures are performed in tertiary $\mathrm{PH}$ centres, ${ }^{83}$ with the experience and familiarity of anaesthetic and ICU teams in dealing with $\mathrm{PH}$ crises and acute RV failure.

Maternal mortality in patients with $\mathrm{PAH}$ has improved in recent years but remains unacceptably high (17\%-33\%). ${ }^{84}$ If a pregnancy progresses, $\mathrm{PAH}$ severity and the obstetric history influence outcome. ${ }^{85}$ Most $\mathrm{PH} /$ maternity units maximise pulmonary vasodilators early in the pregnancy with a prostanoid and PDE5 inhibitor. ${ }^{86}$ Patients undergo regular echocardiography to track RV function. The timing of admission and delivery will be determined by the clinical status of the mother and fetal growth. In those with severe disease, prolonged inpatient stay may be necessary and early elective delivery, often from 34 weeks, balancing maternal risk and the risks of fetal prematurity. Debate continues over optimal anaesthetic regimens, with both regional (combined spinal/epidural) and general anaesthesia used. Meticulous fluid balance with optimisation of RV filling pressures is crucial. Following delivery of the fetoplacental unit, placental autotransfusion (up to $500 \mathrm{~mL}$ ) can dangerously load a failing RV. Drugs that increase PVR, such as Entonox and prostaglandin PGF2 $\alpha$ (hemabate), should be avoided. In patients with cyanosis, postpartum haemorrhage is common and should be managed aggressively. ${ }^{87}$ Many cases of maternal mortality occur in the postpartum period, and almost all patients will require aggressive diuresis and monitoring in ICU for at least 72 hours, with particular reference to fluid balance, RV function and the risk of thrombosis/bleeding. In high-risk patients, extracorporeal membrane oxygenation on standby is recommended.

\section{Suggested approach}

Essential elective surgery should be performed in tertiary $\mathrm{PH}$ centres, with close postoperative haemodynamic monitoring to prevent $\mathrm{PH}$ crises. In a progressing pregnancy, liaison with the $\mathrm{PH}$ centre is recommended, with planned early admission for delivery. In both scenarios, senior $\mathrm{PH}$, anaesthetic and intensive care support is critical.

\section{General comment on resuscitation and end of life care}

Few studies have examined cardiopulmonary resuscitation (CPR) in PAH. Hoeper et al described the experience of physicians across 17 centres in Europe and the US between 1997 and $2002^{49}$ : of 3120 patients with PH treated in these centres, 513 (16\%) had cardiac and respiratory arrest. CPR was attempted in 132 patients and was unsuccessful in 79\% of these. Only eight patients $(6 \%)$ survived at 90 days without neurological deficit, despite $63 \%$ of patients already staying in an ICU at the time of CPR. Of those that survived initial CPR, nearly all had a reversible cause, for example cardiac tamponade post catheter procedure. Sztrymf et al described 46 consecutive patients admitted to ICU with clinical decompensation at a large reference centre in France. $^{21}$ The mean length of stay was 14 days, with an ICU mortality of $41 \%$. Of the 27 patients discharged, six were dead at 3 months and seven required readmission to ICU. Triggers for ICU admission were present in 19 patients and included sepsis, change or unplanned withdrawal of pulmonary vasodilator therapy or diuretics and dysrhythmia. Of note, no patients were intubated during episodes of decompensation, given its dismal association with poor prognosis in patients with $\mathrm{PAH}$.

In practice, management options may be limited in patients with decompensated PAH not on an active transplant list. If already listed for transplant, extracorporeal life support may be a realistic bridge to transplant and close discussions with the relevant transplant centre are key. It may be reasonable, especially in those with advanced $\mathrm{PAH}$, to have an advanced care plan, including a 'do not attempt resuscitation' (DNAR) order. This should be communicated to the GP, ambulance service and emergency departments to avoid futile or inappropriate resuscitation attempts. Palliative care teams in hospital and in the community provide invaluable assistance in clarifying end of life wishes, regular communication and support for the family, as well as review of distressing symptoms including breathlessness. DNAR orders, and indeed decisions not to intubate, are usually appropriate in $\mathrm{PAH}$ patients with a poor prognosis and few ongoing treatment options.

\section{Suggested approach}

Despite the lack of evidence, it would appear reasonable for decompensated patients with PAH to be considered for ICU admission, when reversible causes are present. However, intubation and mechanical ventilation may have adverse effects on RV function, with patients with PAH very unlikely to respond to $\mathrm{CPR}$ in the absence of a reversible cause. It is important that the holistic needs of the patient are considered and that patients are not deprived of supportive and palliative care. 


\section{CONCLUSION}

In this paper, management principles are summarised for the most important emergency scenarios encountered in PAH. Mortality for those requiring level 2 care or above is high, exceeding $40 \%$, and relates to the onset of decompensated RV function. PAH is rare, but more patients are diagnosed and treated early, and are living longer. Understanding the management principles of CRASH scenarios is essential not just for PH teams but for all front-line physicians, with the avoidance of RV failure being crucial in optimising survival. Early decisions regarding treatment escalation to level 2 or 3 care should identify potentially reversible acute conditions, except in some patients listed for transplantation. Early discussions regarding end of life care and escalation planning, between local and specialist $\mathrm{PH}$ teams in advance, with input from palliative care teams, is encouraged at onset.

\section{Author affiliations \\ ${ }^{1}$ National Pulmonary Hypertension Service, Royal Brompton Hospital, London, UK ${ }^{2}$ Adult Congenital Heart Disease Service, Royal Brompton Hospital, London, UK ${ }^{3}$ Department of Respiratory Medicine, Guys and St. Thomas's Hospital, London, UK ${ }^{4}$ National Pulmonary Hypertension Service, Royal Free Hospital, London, UK ${ }^{5}$ National Pulmonary Hypertension Service, Hammersmith Hospital, London, UK ${ }^{6}$ National Pulmonary Hypertension Service, Papworth Hospital, London, UK ${ }^{7}$ National Pulmonary Hypertension Service, Scottish Pulmonary Vascular Unit, Golden Jubilee National Hospital, Cambridge, UK \\ ${ }^{8}$ National Pulmonary Hypertension Service, Freeman Hospital, Newcastle upon Tyne, UK \\ ${ }^{9}$ National Pulmonary Hypertension Service, The Mater Hospital, Dublin, Republic of Ireland \\ ${ }^{10}$ National Pulmonary Hypertension Service, Pulmonary Vascular Unit, Royal Hallamshire Hospital, Sheffield, UK}

Contributors LP, KD, PM, RA, LS, JW wrote sections, and LP and KD compiled the initial draft, acquisitions, analysis, interpretation of all data. All authors revised it critically for important intellectual content. All authors oversaw final approval of the version to be published. All authors had agreement to be accountable for all aspects of the work.

\section{Competing interests None declared.}

Provenance and peer review Not commissioned; externally peer reviewed.

(c) Article author(s) (or their employer(s) unless otherwise stated in the text of the article) 2017. All rights reserved. No commercial use is permitted unless otherwise expressly granted.

\section{REFERENCES}

1 Simonneau G, Gatzoulis MA, Adatia I, et al. Updated clinical classification of pulmonary hypertension. J Am Coll Cardiol 2013;62(25 Suppl):D34-41.

2 Kiely DG, Elliot CA, Sabroe I, et al. Pulmonary hypertension: diagnosis and management. BMJ 2013;346:f2028.

3 Galiè N, Humbert M, Vachiery JL, et al. 2015 ESC/ERS guidelines for the diagnosis and treatment of pulmonary hypertension: the Joint Task Force for the diagnosis and treatment of pulmonary hypertension of the European Society of Cardiology (ESC) and the European Respiratory Society (ERS): Endorsed by: association for European Paediatric and Congenital Cardiology (AEPC), International Society for Heart and Lung Transplantation (ISHLT). Eur Respir J 2015:46:903-75.

4 National Pulmonary Hypertension Centres of the UK and Ireland. Consensus statement on the management of pulmonary hypertension in clinical practice in the UK and Ireland. Thorax 2008;63(Suppl 2):ii1-41.

5 Gibbons Kroeker CA, Adeeb S, Shrive NG, et al. Compression induced by RV pressure overload decreases regional coronary blood flow in anesthetized dogs. Am J Physiol Heart Circ Physiol 2006;290:H2432-8.

6 Vlahakes GJ, Turley K, Hoffman JI. The pathophysiology of failure in acute right ventricular hypertension: hemodynamic and biochemical correlations. Circulation 1981;63:87-95.

7 Galiè N, Saia F, Palazzini M, et al. Left Main coronary artery compression in patients with pulmonary arterial hypertension and Angina. J Am Coll Cardiol 2017;69:2808- 17.

8 Araújo I, Escribano P, Lopez-Gude MJ, et al. Giant pulmonary artery aneurysm in a patient with vasoreactive pulmonary hypertension: a case report. BMC CardiovasC Disord 2011;11:64.

9 Decuypere $\mathrm{V}$, Delcroix $\mathrm{M}$, Budts $\mathrm{W}$. Left main coronary artery and right pulmonary vein compression by a large pulmonary artery aneurysm. Heart 2004;90:21e-21.
10 Sivakumar K, Rajan M, Francis G, et al. Extrinsic compression of the left coronary ostium by the pulmonary trunk: management in a case of eisenmenger syndrome. Tex Heart Inst J 2010;37:95-8.

11 Żyłkowska J, Kurzyna M, Florczyk M, et al. Pulmonary artery dilatation correlates with the risk of unexpected death in chronic arterial or thromboembolic pulmonary hypertension. Chest 2012;142:1406-16.

12 Filusch A, Giannitsis E, Katus HA, et al. High-sensitive troponin T: a novel biomarker for prognosis and disease severity in patients with pulmonary arterial hypertension. Clin Sci 2010;119:207-13.

13 Cohen HA, Baird MG, Rouleau JR, et al. Thallium 201 myocardial imaging in patients with pulmonary hypertension. Circulation 1976;54:790-5.

14 Lee MS, Oyama J, Bhatia R, et al. Left main coronary artery compression from pulmonary artery enlargement due to pulmonary hypertension: a contemporary review and argument for percutaneous revascularization. Catheter Cardiovasc Interv 2010;76:543-50

15 Provencher $S$, Herve $P$, Jais $X$, et al. Deleterious effects of beta-blockers on exercise capacity and hemodynamics in patients with portopulmonary hypertension. Gastroenterology 2006;130:120-6.

16 Kawut SM, Horn EM, Berekashvili KK, et al. New predictors of outcome in idiopathic pulmonary arterial hypertension. Am J Cardiol 2005;95:199-203.

17 Greyson CR. The right ventricle and pulmonary circulation: basic concepts. Rev Esp Cardiol 2010;63:81-95.

18 Gaynor SL, Maniar HS, Bloch JB, et al. Right atrial and ventricular adaptation to chronic right ventricular pressure overload. Circulation 2005;112(9 Suppl):1212-8.

19 Granton J, Mercier O, De Perrot M. Management of severe pulmonary arterial hypertension. Semin Respir Crit Care Med 2013;34:700-13.

20 Hoffman D, Sisto D, Frater RW, et al. Left-to-right ventricular interaction with a noncontracting right ventricle. J Thorac Cardiovasc Surg 1994;107:1496-502.

21 Sztrymf B, Souza R, Bertoletti L, et al. Prognostic factors of acute heart failure in patients with pulmonary arterial hypertension. Eur Respir J 2010;35:1286-93.

22 Bansal S, Lindenfeld J, Schrier RW. Sodium retention in heart failure and cirrhosis: potential role of natriuretic doses of mineralocorticoid antagonist? Circ Heart Fail 2009;2:370-6.

23 Hoeper MM, Granton J. Intensive care unit management of patients with severe pulmonary hypertension and right heart failure. Am J Respir Crit Care Med 2011;184:1114-24.

24 Price LC, Wort SJ, Finney SJ, et al. Pulmonary vascular and right ventricular dysfunction in adult critical care: current and emerging options for management: a systematic literature review. Crit Care 2010;14:R169.

25 Kerbaul F, Rondelet B, Motte $\mathrm{S}$, et al. Effects of norepinephrine and dobutamine on pressure load-induced right ventricular failure. Crit Care Med 2004;32:1035-40.

26 Braun EB, Palin CA, Hogue CW. Vasopressin during spinal anesthesia in a patient with primary pulmonary hypertension treated with intravenous epoprostenol. Anesth Analg 2004;99:36-7.

27 Price LC, Forrest P, Sodhi V, et al. Use of vasopressin after caesarean section in idiopathic pulmonary arterial hypertension. Br J Anaesth 2007;99:552-5.

28 Tayama E, Ueda T, Shojima T, et al. Arginine vasopressin is an ideal drug after cardiac surgery for the management of low systemic vascular resistant hypotension concomitant with pulmonary hypertension. Interact Cardiovasc Thorac Surg 2007:6:715-9.

29 Tisdale JE, Patel R, Webb CR, et al. Electrophysiologic and proarrhythmic effects of intravenous inotropic agents. Prog Cardiovasc Dis 1995;38:167-80.

30 Kerbaul F, Gariboldi V, Giorgi R, et al. Effects of levosimendan on acute pulmonary embolism-induced right ventricular failure. Crit Care Med 2007;35:1948-54.

31 Meryon IC, Marino P. Novel strategies for the management of right ventricular failure with pulmonary hypertension. Thorax 2012;67:368-70.

32 Pitsiou G, Paspala A, Bagalas V, et al. Inhaled iloprost plus levosimendan to decompensate right heart failure due to chronic thromboembolic pulmonary hypertension. Anaesth Intensive Care 2013;41:554-6.

33 Khazin V, Kaufman Y, Zabeeda D, et al. Milrinone and nitric oxide: combined effect on pulmonary artery pressures after cardiopulmonary bypass in children. J Cardiothorac Vasc Anesth 2004;18:156-9.

34 Fukazawa K, Poliac LC, Pretto EA. Rapid assessment and safe management of severe pulmonary hypertension with milrinone during orthotopic liver transplantation. Clin Transplant 2010;24:515-9.

35 Buckley MS, Feldman JP. Nebulized milrinone use in a pulmonary hypertensive crisis Pharmacotherapy 2007;27:1763-6.

36 Tongers J, Schwerdtfeger B, Klein G, et al. Incidence and clinical relevance of supraventricular tachyarrhythmias in pulmonary hypertension. Am Heart $J$ 2007;153:127-32.

37 Ruiz-Cano MJ, Gonzalez-Mansilla A, Escribano P, et al. Clinical implications of supraventricular arrhythmias in patients with severe pulmonary arterial hypertension. Int J Cardiol 2011;146:105-6.

38 Olsson KM, Nickel NP, Tongers J, et al. Atrial flutter and fibrillation in patients with pulmonary hypertension. Int J Cardiol 2013;167:2300-5.

39 Rottlaender D, Motloch LJ, Schmidt D, et al. Clinical impact of atrial fibrillation in patients with pulmonary hypertension. PLoS One 2012;7:e33902. 
40 Medi C, Kalman JM, Ling LH, et al. Atrial electrical and structural remodeling associated with longstanding pulmonary hypertension and right ventricular hypertrophy in humans. J Cardiovasc Electrophysio/ 2012;23:614-20.

41 Daliento L, Somerville J, Presbitero P, et al. Eisenmenger syndrome. Factors relating to deterioration and death. Eur Heart J 1998; 19:1845-55.

42 Diller GP, Dimopoulos K, Broberg CS, et al. Presentation, survival prospects, and predictors of death in Eisenmenger syndrome: a combined retrospective and casecontrol study. Eur Heart J 2006;27:1737-42.

43 Folino AF, Bobbo F, Schiraldi C, et al. Ventricular arrhythmias and autonomic profile in patients with primary pulmonary hypertension. Lung 2003;181:321-8.

44 Lip GY, Nieuwlaat R, Pisters R, et al. Refining clinical risk stratification for predicting stroke and thromboembolism in atrial fibrillation using a novel risk factor-based approach: the euro heart survey on atrial fibrillation. Chest 2010;137:263-72.

45 Showkathali R, Tayebjee MH, Grapsa J, et al. Right atrial flutter isthmus ablation is feasible and results in acute clinical improvement in patients with persistent atrial flutter and severe pulmonary arterial hypertension. Int J Cardiol 2011;149:279-80.

46 Peacock A, Ross K. Pulmonary hypertension: a contraindication to the use of \{beta\}adrenoceptor blocking agents. Thorax 2010;65:454-5.

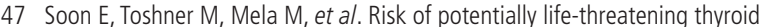
dysfunction due to amiodarone in idiopathic pulmonary arterial hypertension patients. J Am Coll Cardiol 2011;57:997-8.

48 Bradfield J, Shapiro S, Finch W, et al. Catheter ablation of typical atrial flutter in severe pulmonary hypertension. J Cardiovasc Electrophysiol 2012;23:1185-90.

49 Hoeper MM, Galié N, Murali S, et al. Outcome after cardiopulmonary resuscitation in patients with pulmonary arterial hypertension. Am J Respir Crit Care Med 2002;165:341-4.

50 Tzelepis GE, Kelekis NL, Plastiras SC, et al. Pattern and distribution of myocardial fibrosis in systemic sclerosis: a delayed enhanced magnetic resonance imaging study. Arthritis Rheum 2007;56:3827-36

51 Temple ISH, Nicoll R, Griffiths L, et al. Europace Congress. Europace 2013;15(supp 4):iv26-30.

52 Rich JD, Thenappan T, Freed B, et al. QTc prolongation is associated with impaired right ventricular function and predicts mortality in pulmonary hypertension. Int J Cardiol 2013;167:669-76.

53 Rush B, Biagioni BJ, Berger L, et al. Mechanical Ventilation Outcomes in Patients With Pulmonary Hypertension in the United States: A National Retrospective Cohort Analysis. J Intensive Care Med 2016 (Epub ahead of print: 8 Jun 2016).

54 Mermel LA, Allon M, Bouza E, et al. Clinical practice guidelines for the diagnosis and management of intravascular catheter-related infection: 2009 Update by the infectious diseases Society of America. Clin Infect Dis 2009;49:1-45.

55 Boucly A, O'Connell C, Savale L, et al. [Tunnelled central venous line-associated infections in patients with pulmonary arterial hypertension treated with intravenous prostacyclin]. Presse medicale 2016;45:20-8.

56 Habib G, Lancellotti P, Antunes MJ, et al. 2015 ESC guidelines for the management of infective endocarditis: the Task Force for the management of infective endocarditis of the European Society of Cardiology (ESC). Endorsed by: european Association for Cardio-Thoracic Surgery (EACTS), the European Association of Nuclear Medicine (EANM). Eur Heart J 20152015:36:3075-128.

57 Ghofrani HA, D'Armini AM, Grimminger F, et al. Riociguat for the treatment of chronic thromboembolic pulmonary hypertension. N Engl J Med 2013;369:319-29.

58 Pulido T, Adzerikho I, Channick RN, et al. Macitentan and morbidity and mortality in pulmonary arterial hypertension. N Engl J Med 2013;369:809-18.

59 Liang CD, Huang SC, Su WJ, Wj S. Pulmonary vascular obstructive disease and hemoptysis in a child with tetralogy of Fallot and patent ductus arteriosus. $J$ Formos Med Assoc 1997:96:121-4

60 Broberg C, Ujita M, Babu-Narayan S, et al. Massive pulmonary artery thrombosis with haemoptysis in adults with Eisenmenger's syndrome: a clinical dilemma. Heart 2004;90:e63.

61 Cantu J, Wang D, Safdar Z. Clinical implications of haemoptysis in patients with pulmonary arterial hypertension. Int J Clin Pract Supp/ 2012;177:5-12.

62 Reesink HJ, van Delden OM, Kloek JJ, et al. Embolization for hemoptysis in chronic thromboembolic pulmonary hypertension: report of two cases and a review of the literature. Cardiovasc Intervent Radiol 2007:30:136-9.
63 Ghigna MR, Guignabert C, Montani D, et al. BMPR2 mutation status influences bronchial vascular changes in pulmonary arterial hypertension. Eur Respir J 2016;48:1668-81.

64 Tio D, Leter E, Boerrigter B, et al. Risk factors for hemoptysis in idiopathic and hereditary pulmonary arterial hypertension. PLoS One 2013;8:e78132.

65 Prapa M, McCarthy KP, Dimopoulos K, et al. Histopathology of the great vessels in patients with pulmonary arterial hypertension in association with congenital heart disease: large pulmonary arteries matter too. Int J Cardiol 2013;168:2248-54.

66 Roofthooft MT, Douwes JM, Vrijlandt EJ, et al. Frequency and prognostic significance of hemoptysis in pediatric pulmonary arterial hypertension. Am J Cardiol 2013;112:1505-9.

67 Devine MJ, Radford DJ. Treatment of haemoptysis in pulmonary atresia with tranexamic acid. Cardiol Young 2013;23:304-5.

68 Moen CA, Burrell A, Dunning J. Does tranexamic acid stop haemoptysis? Interact Cardiovasc Thorac Surg 2013;17:991-4.

69 Hicks K, Peng D, Gajewski JL. Treatment of diffuse alveolar hemorrhage after allogeneic bone marrow transplant with recombinant factor VIla. Bone Marrow Transplant 2002;30:975-8.

70 Pea L, Roda L, Boussaud V, et al. Desmopressin therapy for massive hemoptysis associated with severe leptospirosis. Am J Respir Crit Care Med 2003;167:726-8.

71 Cantu JA, Safdar Z. Hemoptysis requiring bronchial artery embolization in pulmonary arterial hypertension. South Med J 2010;103:887-91.

72 Petraco R, Dimopoulos K, Rubens M, et al. Bronchial artery embolization for pulmonary arterial hypertension and recurrent hemoptysis? Am J Cardiol 2008;101:1064-5.

73 Brinson GM, Noone PG, Mauro MA, et al. Bronchial artery embolization for the treatment of hemoptysis in patients with cystic fibrosis. Am J Respir Crit Care Med 1998;157(6 Pt 1):1951-8.

74 Chun JY, Morgan R, Belli AM. Radiological management of hemoptysis: a comprehensive review of diagnostic imaging and bronchial arterial embolization. Cardiovasc Intervent Radiol 2010;33:240-50.

75 Żyłkowska J, Kurzyna M, Pietura R, et al. Recurrent hemoptysis: an emerging life-threatening complication in idiopathic pulmonary arterial hypertension. Chest 2011;139:690-3.

76 Lundgren R, Häggmark S, Reiz S. Hemodynamic effects of flexible fiberoptic bronchoscopy performed under topical anesthesia. Chest 1982;82:295-9.

77 Wahidi MM, Rocha AT, Hollingsworth JW, et al. Contraindications and safety of transbronchial lung biopsy via flexible bronchoscopy. Respiration 2005;72:285-95.

78 Dweik RA, Stoller JK. Role of bronchoscopy in massive hemoptysis. Clin Chest Med 1999:20:89-105

79 Ong TH, Eng P. Massive hemoptysis requiring intensive care. Intensive Care Med 2003:29:317-20.

80 Ramakrishna G, Sprung J, Ravi BS, et al. Impact of pulmonary hypertension on the outcomes of noncardiac surgery: predictors of perioperative morbidity and mortality. $J$ Am Coll Cardiol 2005;45:1691-9.

81 Minai OA, Venkateshiah SB, Arroliga AC. Surgical intervention in patients with moderate to severe pulmonary arterial hypertension. Conn Med 2006;70:239-43.

82 Price LC, Montani D, Jaïs X, et al. Noncardiothoracic nonobstetric surgery in mild-tomoderate pulmonary hypertension. Eur Respir J 2010;35:1294-302.

83 Meyer S, McLaughlin VV, Seyfarth HJ, et al. Outcomes of noncardiac, nonobstetric surgery in patients with PAH: an international prospective survey. Eur Respir J 2013:41:1302-7.

84 Bédard E, Dimopoulos K, Gatzoulis MA. Has there been any progress made on pregnancy outcomes among women with pulmonary arterial hypertension? Eur Heart J 2009;30:256-65.

85 Jaïs $X$, Olsson KM, Barbera JA, et al. Pregnancy outcomes in pulmonary arterial hypertension in the modern management era. Eur Respir J 2012;40:881-5.

86 Kiely DG, Condliffe R, Webster V, et al. Improved survival in pregnancy and pulmonary hypertension using a multiprofessional approach. BJOG 2010;117:565-74.

87 Hayman RG, Arulkumaran S, Steer PJ. Uterine compression sutures: surgical management of postpartum hemorrhage. Obstet Gynecol 2002;99:502-6. 